\title{
La construcción social de la "queretaneidad" y sus límites ante la diferenciación social
}

\author{
Rafael Loyola Díaz
}

Ana Díaz Aldret, 2011

La paz y sus sombras. La cultura política en el estado de Querétaro

Miguel Ángel Porrúa, México, 457 pp.

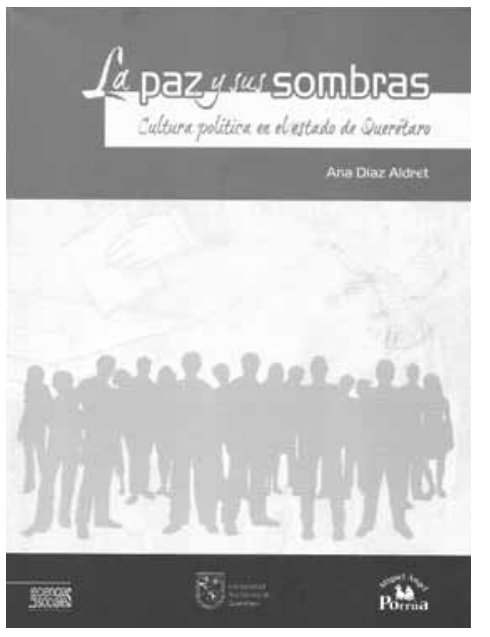

$\mathrm{D}$ e manera no tan distinta a como ha ocurrido en otras entidades de México donde se experimentaron procesos de modernización durante la segunda mitad del siglo pasado - aunque con características particulares que la autora enfatiza a lo largo del libro-, el caso de Querétaro siempre brota por la claridad de la hoja de ruta que siguió para la industrialización y el desarrollo que se le reconocen en la
The Social Construction of "Queretaneidad" and its Limits in the Face of Social Differentiation

Rafael Loyola Díaz: Instituto de Investigaciones Sociales, Universidad Nacional Autónoma de México, Distrito Federal, México loyola@servidor.unam.mx

Desacatos, núm. 39, mayo-agosto 2012, pp. 207-211 actualidad: acuerdo entre las elites para impulsar el cambio, incorporación de capital humano foráneo para fomentar la transformación y asumir nuevas responsabilidades, atracción de capitales externos, inversión en infraestructura, sintonía de los mandos estatales con las políticas del centro - en específico con las de descentralización de los años setenta-, paquete de políticas públicas y de estímulos para la industrialización, visión de largo plazo y continuidad en las acciones.

El éxito de esa estrategia se encuentra en el libro de Ana Díaz Aldret. Es el resultado de una investigación provocadora, con la particularidad de que la autora también es fruto de la migración o de las miradas externas, al igual que las reconocidas aportaciones 
de la historiadora Marta Eugenia García Ugarte, referencia reiterada y uno de los soportes de la obra. El resultado del arduo trabajo de pesquisa de Ana Díaz Aldret es por demás lúcido, riguroso, creativo y propositivo para vislumbrar lo que ha sido la modernización de la entidad durante los últimos 30 años, en perspectiva histórica y con una metodología muy a tono a como debe realizarse la investigación social en la actualidad, esto es con intersecciones disciplinarias entre la antropología social, la sociología y la ciencia política. Desde esta plataforma, la autora contribuye a desentrañar la configuración de la cultura política queretana, explora las claves de una modernización sin mayores fracturas sociales, indaga acerca de los detonantes y de los pasillos que condujeron a la alternancia política y devela la estructuración del espacio público para el despliegue de una cultura política que a regañadientes ha asumido los rudimentos de la democracia en la entidad, como ha ocurrido en otras regiones del país después del pesado dominio priista y que no es tan diferente de los vaivenes $y$ retrocesos que se registran también en el plano federal.

Para emprender su investigación, la autora recurrió a tres bloques. En el primero analiza cómo se construyó una cultura política particular en la entidad, hasta llegar a la alternancia luego de que el estado se insertó en un proceso

acelerado de industrialización sin mayor apertura política que posteriormente dio pie a cierta liberalización, aunque en forma tardía respecto de los cambios políticos a nivel nacional desde finales de la década de 1980 y en relación con otros estados donde se empujó la alternancia, como Chihuahua, Baja California o Nuevo León. En esta circunstancia, Querétaro semejaba más el monolitismo de los estados sureños, con la diferencia de que ahí sí se había experimentado un proceso profundo de transformación social. En el segundo bloque Ana Díaz Aldret analiza la región de la Sierra Gorda, en tanto espacio relativamente autónomo dentro de la vocación autonómica de las elites queretanas. Observa con atención los rasgos particulares de su propia cultura política, la estructura patriarcal y familiar del control político y cómo se introdujo cierta pluralidad política. El tercer bloque es una fotografía de la cultura política queretana, como muestra de cristalización de una comunidad de referentes culturales, valores y sentidos que provienen de la lejana Colonia y que se troquelaron en distintos momentos históricos, siendo tan pesada su factura que hasta los sectores medios y altos que han migrado al estado se han visto en la necesidad de asumirlos y manejarlos para estar a tono con el concierto de armonía y prosperidad del que tanto se enorgullece la queretaneidad.

Tal andamiaje narrativo sirve de escenario para que la autora discurra en la pesquisa de algunas de sus preocupaciones. Antes que nada, le apasiona discernir sobre el origen de la cultura política de la queretaneidad, las fuentes que la nutrieron y los momentos históricos en que se fue procesando, cómo opera en la actualidad, sus fronteras y los resquicios a través de los cuales se han filtrado con resistencias la pluralidad política y los prolegómenos de una democracia que con dificultad acepta la diversidad, la independencia de los actores políticos, la autonomía de la sociedad civil y su derecho a organizarse al margen del Estado para plantear demandas legítimas, así como su institucionalidad para operar como contrapeso y vigilante del poder. Luego se ocupa del tema de la democracia. Le interesa responder por qué se introdujo con retardo en la entidad e indagar sobre el sinuoso camino por el que se ha abierto paso, los espacios limitados de su instrumentación, lo beneficiosa que ha sido para las elites gobernantes y acerca de sus pobres resultados de la mano de un partido, Acción Nacional (PAN), que siempre la había 
vendido como opción para corregir los males ancestrales y las desviaciones del largo dominio priista.

A la autora también le resuena la pregunta de por qué la alternancia política ha abonado poco en la entidad para el desarrollo y consolidación de la democracia, para favorecer la autonomía de la sociedad civil y fomentar la expresión plural, profesional, atrevida e incómoda de la prensa. En resumen, a Ana Díaz Aldret le interesa entender esa predisposición de las elites, lo mismo panistas que priistas, a controlar todo desde el aparato público, a frenar los respiraderos independientes de la sociedad y a mantener su modelo de orden y armonía como requisito básico para continuar con la prosperidad. Finalmente, le apasiona esa microrregión de la Sierra, lugar de autonomía ancestral por sus condiciones geográficas y de pobreza, su relación con el centro político y las Huastecas, las particularidades de su cultura política y los estrechos senderos por donde se fue colando, a cuentagotas, la pluralidad política, proceso que se facilitó hasta que sucedió la alternancia política en el Ejecutivo estatal.

La sorpresa para la autora, y una de sus principales aportaciones, radica en que los gobiernos panistas poco o casi nada hicieron para avanzar en el cambio político del estado, se agiornaron a la cultura priista, mantuvieron y se apoyaron en las estructuras de poder heredadas y conservaron el estado de cosas sin transformaciones mayores. Por lo mismo, las estructuras de dominio permanecieron intactas, se continuó con el recelo, cooptación o represión a las manifestaciones autónomas de la sociedad civil, y se hizo poco, si no es que nada, en materia de profesionalización de los medios de comunicación, a lo que se puede añadir que casi no se avanzó en los ámbitos de transparencia, combate a la corrupción y erradicación del abuso de poder.

En las conclusiones de la autora se puede leer que con la alternancia política el estado de cosas prácticamente siguió igual a como era en las épocas del dominio priista: esto es, vocación por el orden, imposición del control político ante los intentos de autonomía política o social, refracción a lo externo que no se acomode a la estabilidad o a los dictados de las elites, prurito por la limpieza y la conservación rayando en la restauración de viejos referentes culturales y predominio de las relaciones familiares y de amistad sobre las institucionales, así como el aseguramiento de la paz y tranquilidad como requisito para el progreso; además, asfixia de la oposición y del pluralismo político que perturbe el orden $y$, por lo mismo, culto al principio de autoridad y a los resabios de una cultura patriarcal.

Si acaso, donde se marca una diferencia es en los estilos de ejercer el poder. Mientras el Partido Revolucionario Institucional (PRI) es sensible y sabe operar demandas populares - abanico de opciones que va de la atención a la demanda y la cooptación de liderazgos hasta la intimidación y la represión-, parece que al pan se le da fácil el menosprecio de lo popular, se le dificulta negociar y resolver demandas populares, opta por soluciones individuales, de inmediato antepone la ley para contener o desviar peticiones colectivas y autónomas, y es de mecha corta para operar la presión social. En el recuento pormenorizado de Ana Díaz Aldret sobre el conflicto del gobernador Ignacio Loyola con las manifestaciones locales del zapatismo, e incluso con el mismo subcomandante Marcos, llama la atención tanto la desatinada respuesta y reacción del entonces gobernador como las manifestaciones de repudio al desorden y movilizaciones, posición en la que congeniaron tanto priistas como panistas, lo mismo que el sector privado y la jerarquía católica. Como atinadamente advierte la autora, todas las fuerzas vivas y las dos principales formaciones políticas coincidieron en la idea de que a la sociedad queretana no le gusta el desorden y menos cuando viene de fuera. 
No obstante que en Querétaro, como en el resto del país, la experiencia panista ha avanzado poco en materia democrática y en relación con el Estado de derecho, la autora subraya que la alternancia política no dejó de ser saludable para propiciar la escasa pluralidad política que se vive en la entidad. Como ella misma reseña, y de manera no tan distinta a como sucedió en el plano nacional, fueron los grupos de izquierda, los contestatarios, los incómodos para el poder, los que empezaron a pujar y a confrontarse con la dureza del aparato público, pero en los panistas cayeron los beneficios, de forma tal que aprovecharon una crisis en la cuadrilla priista

para hacerse del poder de manera inesperada, proceso que tampoco ha sido diferente en el plano nacional: desde los años sesenta, incluso antes, movimientos de izquierda confrontaron la dureza priista, luego vendría su fractura y la movilización cardenista de finales de los años ochenta con el desaseo y la discusión sobre el supuesto fraude electoral de 1988 , seguido de una dura represión a liderazgos de izquierda, pero fue el PAN el que capitalizó la primera alternancia política.

Sin embargo, esas fisuras en las elites políticas han derivado en una mayor diversidad política, readecuaciones y cambios que han llegado a regiones tan tradicionalmente controladas como las de la Sierra Gorda, que además han permitido márgenes para que sobrevivan organismos civiles como el Grupo Ecológico Sierra Gorda, aunque no sin dificultades con los gobiernos del PAN, poco dispuestos a los organismos autónomos y, quizás, más atentos a su moral religiosa $\mathrm{y}$ a las buenas costumbres que a los desafíos planetarios del medio ambiente.

En este tenor, el libro de Ana Díaz Aldret refresca y enriquece el análisis no sólo para entender el proceso de modernización en Querétaro, sino para vislumbrar y revalorar el papel del Estado en la definición de proyectos de desarrollo, para impulsar modelos económicos y crear entornos institucionales favorables. Asimismo, aporta mucho para visualizar la importancia del acuerdo entre las elites en la promoción y procesamiento de iniciativas de cambio social, y respecto del valor de la continuidad de los esfuerzos para cristalizar transformaciones de gran calado.

Agrego algunos comentarios que pueden servir para futuras indagaciones. En primer lugar, habría que desconfiar de la pregonada tersura con la que se dio en la entidad el proceso de industrialización. Si bien la autora insiste en el control político y en la represión ejercida en algunos momentos, acciones que identifica más selectivas que recurrentes, estimo que todavía habría que indagar en la violencia acaecida en las zonas rurales por el control de tierras y en las acciones soterradas para asegurar el control político. En segundo lugar, todavía existe tela de donde cortar para explorar sobre las condiciones y los factores que han troquelado la cultura política queretana de orden y progreso, particularmente en torno de los mecanismos de reproducción y adaptación de esa cultura, en el entendido de que los medios de comunicación destacan por su falta de profesionalismo, de que los liderazgos intelectuales son frágiles para actualizar el discurso y responder a una sociedad más compleja y con una nueva agenda de pendientes y de que la Iglesia mantiene su presencia, pero en un contexto social cada vez más secularizado, autónomo y diverso.

Inquieta el tema de la democracia desde la perspectiva de que a menudo se confunde la instrumentación de la democracia con el análisis político, de tal suerte que antes de estudiársele desde las entrañas del poder se le analiza en función de si se cumple o no la agenda de la democratización. Esta desviación o frecuente inclinación suele llevar a subrayar el estancamiento democrático y a debatir acerca del incumplimiento de la agenda o de la falta de inclinación de los actores políticos hacia la democracia, dejando de lado el dilema de las transformaciones en las estructuras de poder, de las 
motivaciones de los actores políticos o de las limitaciones o resistencias para avanzar hacia mayores y mejores libertades políticas. Dicho brevemente, con o sin democracia las elites siguen ejerciendo el poder.

Propondría atender más la dinámica de los fenómenos sociales que las teorizaciones sobre los mismos. Las referencias teóricas facilitan la comprensión del fenómeno $y$ ofrecen herramientas de análisis, pero no necesariamente son la clave para lograr nuevos descubrimientos, aparte de que en ocasiones pueden hacer pesada la lectura y alejar a lectores más interesados en conocer el fenómeno que las herramientas de análisis. Para terminar, mencionaré rápidamente algunos temas de investigación que nos sugiere el libro La paz y las sombras. En primer lugar, habría que preguntarse por qué las elites queretanas, las de viejo cuño, se han mantenido en el renglón comercial y de los servicios, además de dominar en el sector agropecuario. La interrogante es: ¿por qué no han trascendido al sector industrial? Salvo la huella y la visión de don Roberto Ruiz Obregón y de otros más, la tentación industrial e innovadora no ha seducido a los empresarios locales, como ha ocurrido en otras regiones.

En esta misma dirección cabe indagar qué pasa con la clase política local. Es de todos reconocido el peso de personalidades de relieve nacional desde la época del polémico Saturnino Osornio, pasando por don Agapito Pozo y políticos destacados que han ocupado la dirigencia nacional del PRI, hasta el caso de que uno de ellos llegó a rozar la presidencia de la república en ocasión de la crisis provocada por el asesinato de Luis Donaldo Colosio en 1994. Llama la atención que los políticos queretanos no se hayan constituido como un grupo de peso, como ha sucedido en otros estados - por ejemplo, el poderoso grupo del Estado de México-, teniendo tras de sí un exitoso experimento de modernización y la fortaleza de la economía de la entidad. Quizá aquí se manifiesta lo que observó Guillermo Boils cuando estudió la arquitectura queretana: las clases altas son discretas, construyen muros altos para no mostrar la riqueza. Igual sucedería con los políticos: llegan hasta cierto punto y no más allá, pues la fortaleza está en el crecimiento económico.

Otro tema que resulta atractivo es el de la coyuntura de 1997, cuando se materializó la alternancia en la entidad. La autora hace alusión a una crisis no sólo en el partido, sino hasta en las familias de los dos contendientes del saliente oficialismo gobernante. Cabe preguntarse qué peso tuvo el revuelo político nacional ocasionado por el asesinato de Colosio, los dichos de que Fernando Ortiz Arana - político queretano de altos vuelos y exdirigente del PRI- estuvo entre las opciones para el remplazo del candidato asesinado, la arrogancia que mostró como candidato a la gubernatura del estado con un cuartel general integrado básicamente por foráneos o el exceso de seguridad que exhibió para apropiarse de la gubernatura estatal, como si ya la tuviera en la bolsa, descuidando el peso y los intereses de los grupos locales y desconociendo que la política de la entidad estaba cambiando. Por último, habría que interrogarse qué tanto puede durar una política de control y de exclusión de las autonomías, al igual que el culto de lo local sobre lo foráneo, en un país en pleno proceso de transformación, ante la creciente violencia y con una nueva agenda que excede por mucho los parámetros regionales. El libro que nos ocupa da luces sobre el significado de la modernización en el estado de Querétaro, sobre la recomposición de las elites políticas, sobre la larga permanencia de una cultura política con características particulares y sobre el estado de la relación entre sociedad y política. Además, es un llamado para profundizar la reflexión, establecer comparativos de procesos similares con otras entidades y para analizar la vigencia de la queretaneidad en una región y un país en plena mutación. 